\title{
Pre-eclampsia is associated with the failure of melanoma cell adhesion molecule (MCAM/ CD146) expression by intermediate trophoblast
}

\author{
Qin Liu ${ }^{1,2}$, Xiyun Yan $^{1}$, Yang $\mathrm{Li}^{3}$, Ying Zhang ${ }^{1}$, Xingang Zhao ${ }^{1}$ and Yi Shen ${ }^{1}$ \\ ${ }^{1}$ Center of Molecular Biology, Institute of Biophysics, Chinese Academy of Sciences, Beijing, PR China; ${ }^{2}$ State \\ Key Laboratory of Microbial Resources, Institute of Microbiology, Chinese Academy of Sciences, Beijing, PR \\ China and ${ }^{3}$ Beijing Obstetrics and Gynecology Hospital, Beijing, PR China
}

\begin{abstract}
The purpose of this study was to determine whether the expression of melanoma cell adhesion molecule (MCAM/CD146), like most other endothelial adhesion molecules, is reduced in the pregnancy disorder preeclampsia, and whether it is associated with the invasiveness of trophoblast. We used immunohistochemical approaches and analyzed 42 placentas from control and pre-eclamptic patients using an anti-CD146 monoclonal antibody. Our data show that in normal placentas, CD146 staining was specifically detected in intermediate trophoblasts that are most invasive and migratory, but not detected in noninvasive cytotrophoblasts or syncytiotrophoblasts. However in pre-eclampsia, CD146 was no longer present in the intermediate trophoblasts, although it was detectable in the blood vessels. At the same time, we tested CD31/PECAM-1, another endothelial adhesion molecule, and found its negative staining for all kinds of trophoblasts in both normal and pre-eclamptic placentas. The different staining patterns of CD146 in the normal and pre-eclamptic placentas provide the first evidence that in pre-eclampsia, intermediate trophoblasts fail to express CD146, implicating that CD146 plays an important role in trophoblast invasion.
\end{abstract}

Laboratory Investigation (2004) 84, 221-228, advance online publication, 22 December 2003; doi:10.1038/labinvest.3700033

Keywords: melanoma cell adhesion molecule; CD146; pre-eclampsia; intermediate trophoblast; CD31

Pre-eclampsia, which often occurs in the second and the third trimesters of pregnancy, is a serious disease and is variously estimated to affect $7-10 \%$ of all pregnancies. Pre-eclampsia is typically characterized by hypertension, proteinuria and edema. In some cases, the fetus stops growing, which leads to intrauterine growth retardation. ${ }^{1}$

Although the etiology of pre-eclampsia is unknown, numerous studies have shown that shallow trophoblast invasion into the spiral arteries and endothelial cell dysfunction are two key features in the pathogenesis of pre-eclampsia. ${ }^{2,3}$ During normal pregnancy, the stem cell-like cytotrophoblasts differentiate directly into syncytiotrophoblasts that synthesize and secrete a variety

Correspondence: Xiyun Yan, Center of Molecular Biology, Institute of Biophysics, Chinese Academy of Sciences, Beijing 100101, PR China.

E-mail: yanxy@sun5.ibp.ac.cn

Received 17 September 2003; revised 22 October 2003; accepted 22 October 2003; published online 22 December 2003 of pregnancy-associated hormones, and into intermediate trophoblasts that are highly invasive and migratory. ${ }^{4}$ Intermediate trophoblasts invade the decidualized endometrium, myometrium and subsequently invade maternal spiral arteries, where the endothelial cells are replaced by the endovascular trophoblasts (Figure 1). This remodeling of the uterine spiral arteries results in the diameter of the spiral arterioles increasing dramatically to form a low-resistance arteriolar system and allow an increasing blood flow to the placenta to keep pace with fetal growth. In the final stage, the endothelial lining is reconstituted. ${ }^{5}$

In pre-eclampsia, the differentiation of cytotrophoblasts to intermediate trophoblasts is incomplete and the intermediate trophoblast invasion is shallow. Endovascular invasion does not proceed beyond the superficial portions of the uterine spiral arterioles. Thus, these spiral arterioles retain their endothelial linings and remain relatively narrow-bore, high-resistance vessels, resulting 


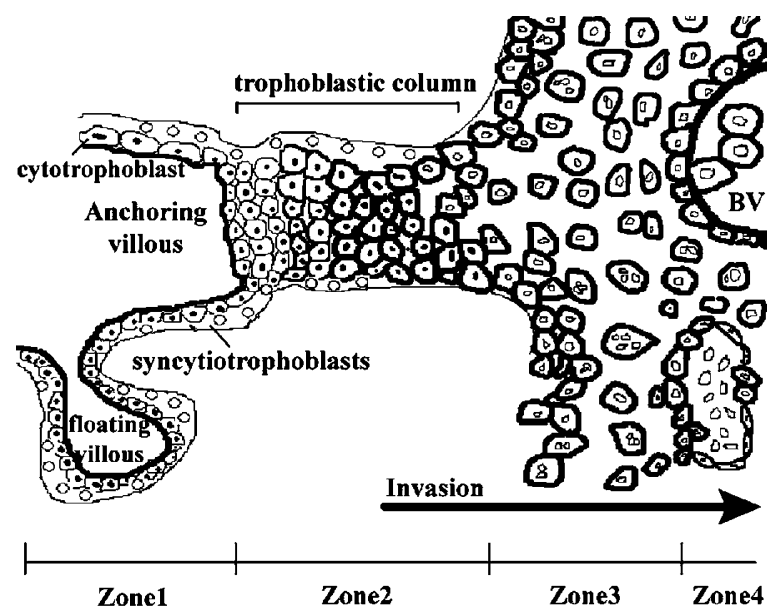

Figure 1 Diagram of the fetal-maternal interface. The interface is demarcated into four zones according to the differentiation of cytotrophoblasts along the invasive pathway. In Zone 1, the stem cytotrophoblasts of anchoring villus and floating villus differentiate directly into syncytiotrophoblasts on the villous surface; but in Zone 2, the cytotrophoblasts of anchoring villous form the trophoblastic column and differentiate gradually into intermediate trophoblasts. The intermediate trophoblasts migrate from the trophoblastic column to form the trophoblastic shell and invade the maternal endometrium (Zone 3) and maternal vasculature (Zone 4) in which the vascular endothelial cells are replaced by intermediate trophoblasts. During the invasion process, part of the intermediate trophoblasts fuse and become multinucleated intermediate trophoblasts. BV, blood vessels.

in the flow of maternal blood to the fetal-placental unit being significantly reduced relative to normal pregnancy. This poor placental perfusion is considered as an important risk factor for developing pre-eclampsia. ${ }^{3}$

The invasive phenotype of trophoblasts is similar to that displayed by malignant tumor cells, which is highly dependent on the appropriate expression of adhesion molecules on the cell surface for anchoring and migration. ${ }^{6}$ The failure of cytotrophoblasts to express properly many of the adhesion molecules is indicative of a deficit in cytotrophoblast differentiation and invasion. Zhou et $a l^{7,8}$ have demonstrated that pre-eclampsia is associated with the lack of cytotrophoblasts to express adhesion molecules including $\alpha \mathrm{v} \beta 3$ integrins, VE-cadherin, VCAM-1 and PECAM-1, which are normally expressed on the vascular endothelium, and the failure to mimic a vascular adhesion phenotype.

The purpose of this study was to determine whether the expression of melanoma cell adhesion molecule (MCAM/CD146), like most of the other endothelial adhesion molecules, is reduced in the placentas complicated by pre-eclampsia. The CD146, also named Mel-CAM, MUC18, ${ }^{9}$ A32 antigen $^{10}$ and S-Endo- $1,{ }^{11}$ is a novel member of the immunoglobulin gene superfamily and contains five extracellular Ig-like domains (V-V-C2-C2-C2), one transmembrane region and a short cytoplasmic tail. ${ }^{9}$
CD146 is only detected in a relatively limited spectrum of normal tissues and tumors, mainly in melanoma, vascular endothelial cells, smooth muscle, implantation site intermediate trophoblast and activated T lymphocytes. ${ }^{9-14}$

CD146 is expressed in more than $90 \%$ of cutaneous melanomas and its expression level is associated with the invasive and metastatic potential of melanoma cells. ${ }^{10,15}$ Increased CD146 expression in melanoma cells promotes tumor growth and metastasis $^{16}$ and decreased CD146 expression reduces tumorigenicity. ${ }^{17}$

CD146 not only takes part in interendothelial interactions as a $\mathrm{Ca}(2+)$-independent cell adhesion molecule, ${ }^{18}$ but also participates in the outside-in signaling that induces phosphorylation of focal adhesion kinase (FAK) and paxillin, and a $\mathrm{Ca}(2+)$ influx, which is closely associated with endothelial cell migration. ${ }^{19,20}$ Recently, we found that CD146, as an active endothelial marker, was involved in angiogenesis, which plays an important role in tumor growth and metastasis. ${ }^{21}$

Shi and co-workers have demonstrated that CD146 is specifically and strongly expressed on human intermediate trophoblasts and trophoblastic tumors, ${ }^{13}$ and regulates the migration of intermediate trophoblasts on smooth muscle cells. ${ }^{22}$ However, it is unknown whether CD146 molecule is expressed in pre-eclamptic placentas in which trophoblast invasion is shallow. To answer this question, we used immunohistochemical approaches and analyzed the expression of the CD146 in the placentas from control and preeclamptic patients.

\section{Materials and methods}

\section{Placental Sources}

A total of 42 human placenta samples were obtained from Beijing Obstetrics and Gynecology Hospital, including 20 third-trimester placentas from preeclamptic patients, 16 third-trimester and 6 firstand second-trimester placentas from the women undergoing termination of apparently normal pregnancy.

\section{Antibodies}

The primary antibodies used were mouse antihuman CD146 monoclonal antibody (mAb) AA98, ${ }^{21}$ rabbit anti-human cytokeratin (CK) polyclonal antibody (Santa Cruz Cooperation, CA, USA) and mouse anti-human CD31 antibody (Cymbus Biotechnology Ltd, Chandlers Ford, UK). Corresponding species-specific Cy3-conjugated (Sigma Chemical Co. Ltd, St Louis, MO, USA) and FITCconjugated (Jackson ImmunoResearch Labs, Inc., Baltimore, MD, USA) secondary antibodies were used. 


\section{Immunohistochemistry}

Fresh tissues were cryosectioned $(10 \mu \mathrm{m})$, fixed in acetone at $4^{\circ} \mathrm{C}$ for $5 \mathrm{~min}$ and then preincubated with $0.3 \% \mathrm{H}_{2} \mathrm{O}_{2}$ in methanol for $30 \mathrm{~min}$ to quench endogenous peroxidases. The sections were blocked with $5 \%$ normal goat serum for $1 \mathrm{~h}$, and incubated with the primary antibody at $4^{\circ} \mathrm{C}$ overnight. For the negative controls, the primary antibody was omitted. The immunoreactivity was revealed using the avidin-biotin-complex peroxidase method.

\section{Double Indirect Immunofluorescence Staining}

Double indirect immunofluorescence staining was preceded as described previously. ${ }^{7}$ Briefly, the sections were blocked with 5\% normal sheep and goat serum for $30 \mathrm{~min}$ and then incubated with two primary antibodies, rabbit anti-cytokeratin antibody and mouse anti-CD146 antibody (or mouse antiCD31 antibody), overnight at $4^{\circ} \mathrm{C}$. For negative controls, the primary antibodies were replaced by PBS. Next, the sections were incubated $60 \mathrm{~min}$ at $37^{\circ} \mathrm{C}$ with two secondary antibodies, Cy3-conjugated sheep anti-mouse IgG and FITC-conjugated goat anti-rabbit IgG. After every incubation step, the sections were washed with PBS. The sections were finally examined under a microscope Olympus RX71-22-000 and photographs were taken using a cooled digital imaging camera KX14-1 under the same exposure conditions without adjusting brightness and contrast.

\section{Quantitative Analysis}

The quantitative analysis was carried out using sections of double indirect immunofluorescence staining, in which anti-cytokeratin- and antiCD146-labeled images were captured on the same field at two different excitation wavelengths. At first, we determined the location of the intermediate trophoblasts in the anti-CD146-labeled images using the anti-cytokeratin-labeled images as a guide. We then calculated the total of the grayscale values of intermediate trophoblasts in the anti-CD146 image. Dividing the grayscale values by the total number of pixels composed of intermediate trophoblasts, the mean of the grayscale was acquired. The mean grayscale of CD146 staining was acquired, after subtracting the background of the negative sample.

Scoring was conducted independently by two observers (LQ and ZY) 'blinded' to the tissue identify. As there were no significant differences between the two sets of scores (ANOVA; $P>0.05$ for all comparisons), the scores of one observer (LQ) were used for subsequent analysis. Scoring was conducted three times independently by LQ. As there were no significant differences among the three sets of scores (ANOVA; $P>0.05$ for all comparisons), one set of results was used for subsequent analysis.

\section{Results}

\section{Study Participants}

The clinical details for the women who provided the third-trimester placentas for this study are summarized in Table 1.

\section{Immunohistochemical Localization of CD146 in Normal Placentas}

To detect the expression of CD146 at the maternalfetal interface in the normal pregnancy, immunohistochemistry was performed. On the maternal side, CD146 staining was restricted in endometrial vessels (Figure 2a), but not on glandular epithelial cells that were recognized by anti-cytokeratin (Figure 2b). Additionally, the decidual cells exhibited faint staining (Figure 2a), which was further confirmed by the indirect immunofluorescence (data not shown). On the fetal side, we found that CD146 was specifically immunostained in the

Table 1 Clinical details for placental immunohistochemistry studies

Control $(\mathrm{n}=16) \quad P E(\mathrm{n}=20)$

$\begin{array}{lrc}\text { Age (years) } & 28.44 \pm 2.58 & 28.05 \pm 4.02 \\ \text { Gestational age at delivery (weeks) } & 31.87 \pm 1.89 & 31.15 \pm 1.34 \\ \text { Birth weight (kg) } & 2.09 \pm 0.18 & 1.26 \pm 0.20^{*} \\ \text { Systolic BP (mmHg) } & 109.4 \pm 7.72 & 161.8 \pm 11.03^{*} \\ \text { Diastolic BP (mmHg) } & 72.5 \pm 6.58 & 112.2 \pm 10.05^{*} \\ \text { Uric protein (g/24 h) } & - & 8.94 \pm 2.80 \\ \text { Edema } & - & \pm-+++\end{array}$

Values are shown as mean \pm s.d.

${ }^{*} P<0.001$, compared with the control pregnant group.

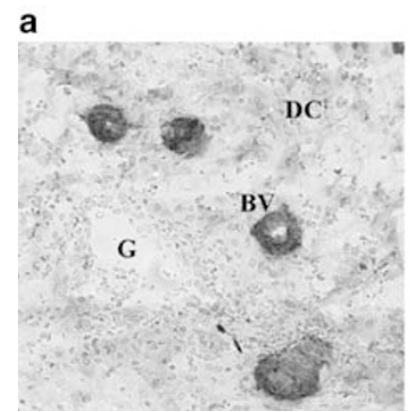

b

Figure 2 CD146 expression is analyzed in the maternal decidua of the normal early-stage pregnancy ( 6 weeks). Immunohistochemistry was performed on frozen sections separately with antiCD146 antibody AA98 $(\mathbf{a} ; \times 200)$ and anti-cytokeratin $(\mathrm{CK})$ to identify all epithelial cells $(\mathbf{b} ; \times 200)$. CD146 staining was intense in the decidual blood vessels (BV; a), but not in the gland (G) recognized by anti-cytokeratin (b). Additionally, the decidual cells (DC) exhibited faint staining (a). 
a

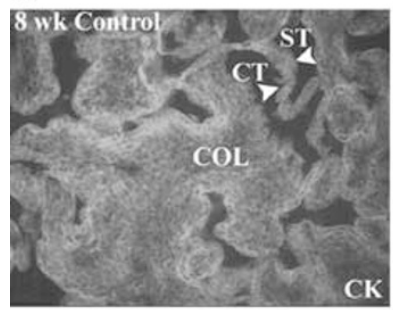

d

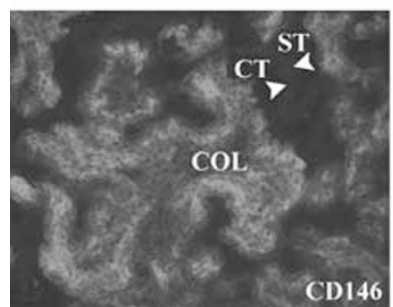

b

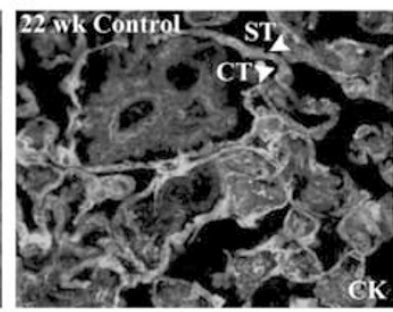

e

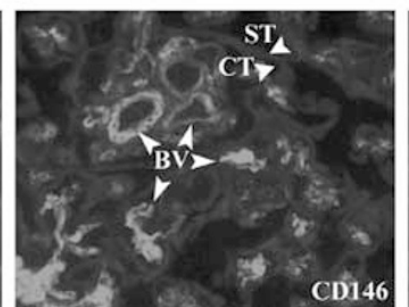

c

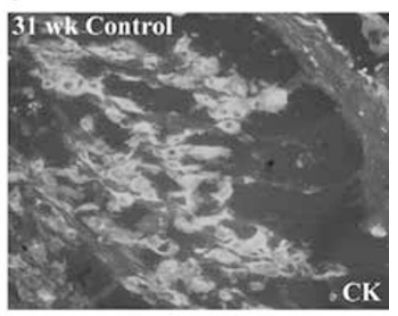

f

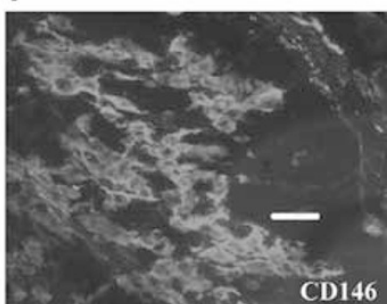

Figure 3 CD146 expression is analyzed in normal placentas through all three trimesters of pregnancy. Frozen sections were double stained with anti-CD146 antibody AA98 $(\mathbf{d}-\mathbf{f} ; \times 200)$ and anti-cytokeratin $(\mathrm{CK})$ to identify all trophoblast populations $(\mathbf{a}-\mathbf{c}$; $\times 200)$. CD146 staining was intense in the intermediate trophoblasts during all three trimesters of pregnancy (ie, 8, 22 and 31 weeks of gestation), but not in syncytiotrophoblasts (ST) or in cytotrophoblasts (CT) of anchoring villus (a and d) and floating villus in which CD146 staining was detectable in uteroplacental blood vessels (BV; b and e). The intensity of CD146 staining on the intermediate trophoblasts was uniformly strong in the trophoblast column (COL) of anchoring villus (d) and in the decidua (f). The scale bar stood for $100 \mu \mathrm{m}$.

a

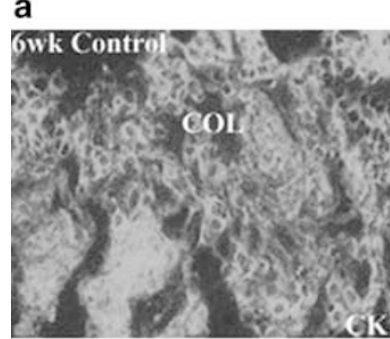

d

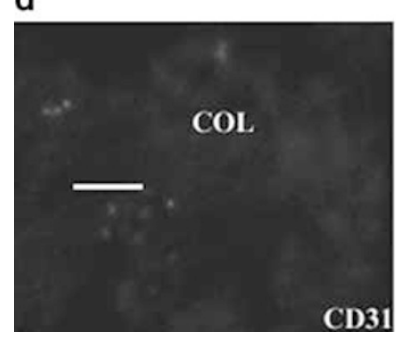

b

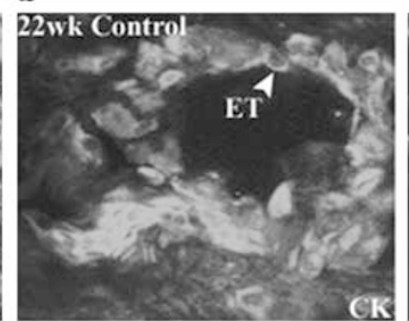

e

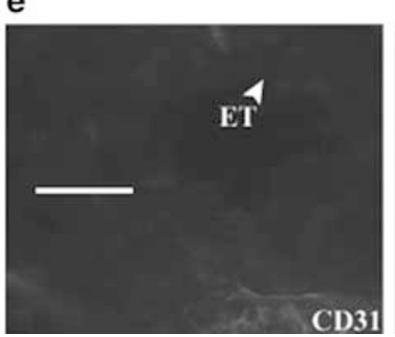

C

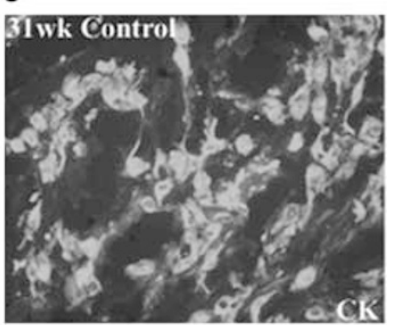

f

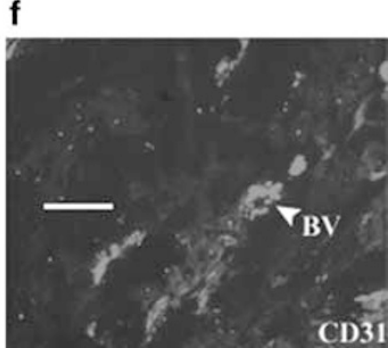

Figure 4 CD31/PECAM-1 expression is analyzed in normal placentas through all three trimesters of pregnancy. Frozen sections were double stained with anti-CD31 (d-f) and anti-cytokeratin (CK) to identify trophoblasts (a-c). The anti-CD31 staining was absent in the intermediate trophoblasts during all three trimesters of pregnancy (ie, 6, 22 and 31 weeks of gestation), both in the trophoblast column $(\mathrm{COL})(\mathbf{d} ; \times 200)$, and in decidua $(\mathbf{f} ; \times 200)$. Although CD31 positive staining for blood vessels $(\mathrm{BV})$ was seen in the decidua (f; arrow pointed), it was not detectable in the blood vessels whose endothelium lining had been replaced by endovascular trophoblast (ET) in the second trimester $(\mathbf{e} ; \times 400)$, whereas these endovascular trophoblasts were recognized by anti-cytokeratin (b). The scale bars stood for $100 \mu \mathrm{m}$.

intermediate trophoblasts, not only in uterine decidua in all three trimesters of normal placentas $(n=22$, Figure $3 \mathrm{f})$ but also in the trophoblastic column of anchoring villus (Figure 3d), whereas CD146 staining was not detected on syncytiotrophoblasts or cytotrophoblasts of anchoring villus (Figure $3 \mathrm{a}$ and $\mathrm{d}$ ) and floating villus (Figure $3 \mathrm{~b}$ and e) in which CD146-positive staining was only detected in villous blood vessels. The intensity of CD146 staining on the extravillous intermediate trophoblasts was uniformly strong, independent of the depth of IT invasion in the uterus and gestation age. In contrast to CD146, CD31/PECAM-1, an endothelial cell adhesion molecule, was not stained in the intermediate trophoblasts in all three trimesters (Figure 4). Although CD31-positive staining for 
blood vessels was seen in decidua (Figure 4f), it was not detectable in the blood vessels whose endothelium lining had been replaced by endovascular trophoblast cells in the second trimester (Figure 4e), whereas these endovascular trophoblasts were recognized by anti-cytokeratin (Figure 4b). The expression of CD146 and CD31 at the maternal-fetal interface in the normal pregnancy is presented in Table 2.

\section{Immunohistochemical Localization of CD146 in Pre- eclamptic Placentas}

In contrast to normal placentas, the immunostaining of anti-CD146 in the pre-eclamptic placentas $(n=20)$ was remarkably reduced or undetectable. CD146 was not only absent from the villous

Table 2 CD146 localization in structures at the maternal-fetal interface

\begin{tabular}{lcc}
\hline Structure & CD146 expression CD31 expression \\
\hline Endometrium & & \\
$\quad$ Gland epithelial cells & - & - \\
$\quad$ Stromal & + & ++ \\
$\quad$ Small vessels & +++ & - \\
Placenta & - & - \\
$\quad$ Villous cytotrophoblast & - & - \\
$\quad$ Villous syncytiotrophoblast & +++ & +++ \\
Intermediate trophoblast & +++ & \\
Villous vessels & & \\
\hline
\end{tabular}

-, Negative; +, faint staining; +++, strong staining. cytotrophoblasts and syncytiotrophoblasts (Figure $5 \mathrm{a}$ and e) but also lacking from intermediate trophoblasts in trophoblastic column (Figure 5c and g), basal plate (Figure 5a and e, b and f) and maternal blood vessels (Figure 5d and h). CD146 was lacking from most intermediate trophoblasts, independent of their location in the uterus. Additionally, we observed two abnormal phenomena in the pre-eclamptic placentas. First, the trophoblastic columns were still observed in the third trimester (Figure 5c), which normally disappear at this stage. Second, the endovascular trophoblast lining was still seen in the maternal blood vessels (Figure 5d), which should have been re-endothelized in the normal third-trimester placentas. At the same time, we tested the CD31 expression in the pre-eclamptic placentas using anti-CD31 antibody. The staining pattern was CD31-negative for trophoblasts and positive for blood vessels (Figure 6), as it was in normal pregnancy.

\section{Quantitative Analysis}

We compared the immunofluorescence staining intensity of CD146 in pre-eclamptic placentas with that in normal placentas of the third trimester using grayscale analysis. As shown in Figure 7, CD146 was highly expressed by intermediate trophoblasts in normal placentas and the mean grayscale $(n=11)$ was $25.766 \pm 3.284$ (mean \pm s.e.). However, the expression of CD146 was lacking in the intermediate trophoblasts in pre-eclamptic placentas. The mean grayscale $(n=20)$ was $3.421 \pm 0.618$. The difference a

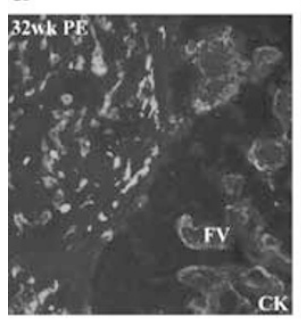

e

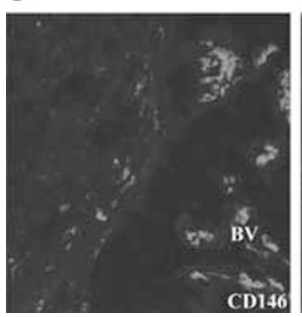

b

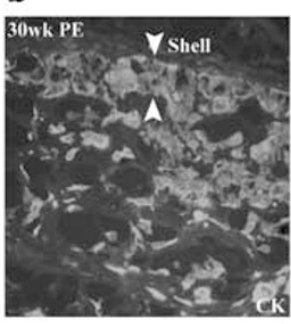

f

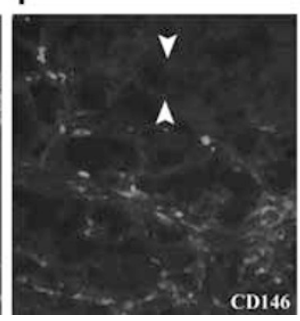

C

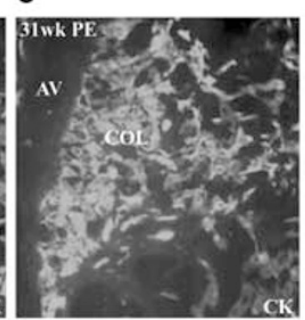

g

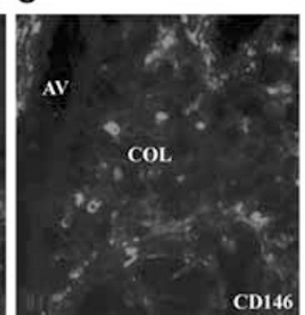

d

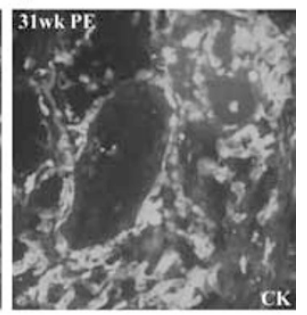

h

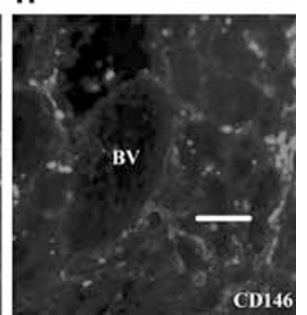

Figure 5 CD146 expression is analyzed in pre-eclamptic third-trimester placentas. Frozen sections were double stained with anticytokeratin (CK) to identify trophoblasts $(\mathbf{a}-\mathbf{d} ; \times 200)$, and anti-CD146 $(\mathbf{e}-\mathbf{h} ; \times 200)$. Although CD146 was present in the blood vessels (BV) of floating villous (FV; e), it is absent in the intermediate trophoblasts in the basal plate (e). The intermediate trophoblasts were lacking anti-CD146 staining whether in the trophoblastic columns (COL; g), trophoblastic shell (shell; f; arrow pointed) of the superficial endometrium, or in maternal blood vessels of deep endometrium (h). Notably, trophoblastic columns of anchoring villus (AV; c) and interrupted blood vessels by trophoblasts (d) were not observed in the normal third-trimester placentas. The scale bar stood for $100 \mu \mathrm{m}$. 


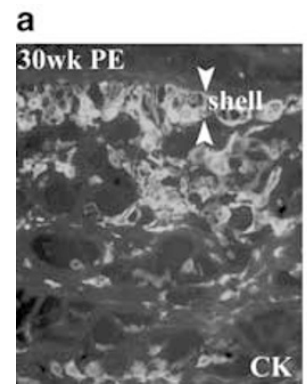

b

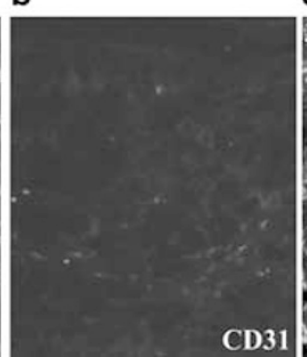

C

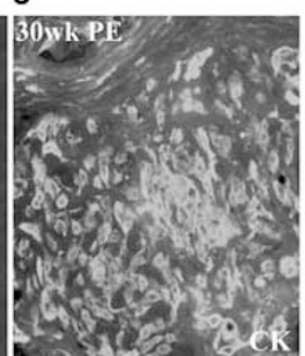

d

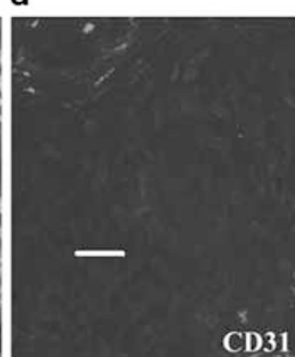

Figure 6 CD31 expression is analyzed in pre-eclamptic third-trimester placentas. Frozen sections were double stained with anticytokeratin (CK) to identify trophoblasts $(\mathbf{a}, \mathbf{c} ; \times 200)$, and anti-CD31 (b, d; $\times 200)$. Anti-CD31 staining was absent in the intermediate trophoblasts in trophoblastic shell (shell) of the basal plate (a and b), and in decidua (c and d), as it did in the normal pregnancy. The scale bar stood for $100 \mu \mathrm{m}$.

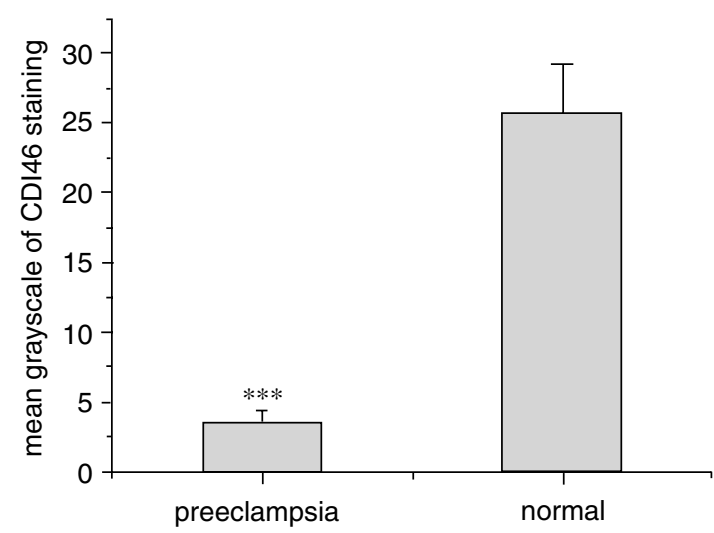

Figure 7 Immunofluorescence staining intensity of CD146 is analyzed in pre-eclamptic placentas and in normal placentas of the third trimester using grayscale analysis. The mean grayscale of CD146 staining in normal placentas $(n=11)$ was $25.766 \pm 3.284$ (mean \pm s.e.), whereas that in pre-eclamptic placentas $(n=20)$ was $3.421 \pm 0.618$. The difference was significant $(P<0.001)$.

of CD146 staining intensity between normal and pre-eclamptic placentas was significant $(P<0.001)$.

\section{Discussion}

This is the first study to examine the expression of CD146 in pregnancy complicated by pre-eclampsia. From carefully selected more than 40 cases of preeclampsia and control pregnancy, we found that in the normal placentas, adhesion molecule CD146 was specifically detected on intermediate trophoblasts in both anchoring villus and decidua through all pregnant stages, whereas villous cytotrophoblast and syncytiotrophoblast were negative. However, the CD146 staining was dramatically reduced or undetectable in pre-eclampsia. From this expression pattern, implications for the role of CD146 in invasiveness can be discussed as follow.

In the control placentas, anti-CD146 antibody AA98 could differentiate intermediate trophoblasts from cytotrophoblasts and syncytiotrophoblasts, which are difficult to distinguish by conventional light microscopy. Our observations have confirmed and extended those reported by Shih et al. ${ }^{13}$ The cytotrophoblasts are characterized as small, undifferentiated and mononucleate stem cells that display proliferative activity consistent with their germinative role. The syncytiotrophoblasts are terminally differentiated cells responsible for the synthesis of a wide variety of steroid and protein hormones. The intermediate trophoblasts, in contrast to the above two subpopulations, are larger and more polyhedral cells with most highly invasive and migratory, also referenced as invasive cytotrophoblast. ${ }^{4}$ The specific expression of CD146 on intermediate trophoblasts can be a useful marker for distinguishing intermediate trophoblasts from the other two trophoblast populations based on both their morphologic features and immunophenotype. In addition, this finding suggests that the expression of CD146 is closely associated with the differentiation and acquired invasive phenotype of cytotrophoblasts.

Furthermore, here we provide the first evidence that the expression of CD146 is dramatically reduced or not detectable anymore on intermediate trophoblasts in pre-eclampsia. This finding supports that CD146 molecule plays significant roles in the process by which cytotrophoblasts differentiate into intermediate trophoblasts and invade the uterus and its arteries where the endothelium is replaced by endovascular trophoblasts. It has been shown that in pre-eclampsia, trophoblast invasion is abnormal and limited to the superficial deciduas. ${ }^{23}$ The two abnormal phenomena we have observed in preeclampsia support that an aberrant trophoblast migration exists in pre-eclampsia. First, the trophoblast columns of anchoring villus were still present in the third trimester, which should disappear at this stage. Second, the endovascular trophoblast lining was still seen in the maternal blood vessels, which should be re-endothelized in normal thirdtrimester placentas. ${ }^{5}$ The abnormal invasion in preeclampsia is often accompanied by a marked change in the expression of cell adhesion molecules on the cell surface. Zhou et $a l^{7,8}$ have reported that many endothelial adhesion molecules, such as $\alpha \mathrm{v} \beta 3$ integrin, VE-cadherin, VCAM-1 and PECAM-1, are upregulated on invasive cytotrophoblast in normal 
placentas but fail to do so in pre-eclampsia. Our data provide further evidence to support the view that pre-eclampsia is a result of abnormal cytotrophoblast differentiation that they do not display the optimum adhesion phenotype for invasion. ${ }^{8}$ Together with the results from previous studies that CD146 is specifically expressed in melanoma cells and its expression level is closely associated with the invasive and metastatic potential of melanoma cells, ${ }^{10,15}$ the findings in this study strongly suggest that CD146 plays an important role in the invasiveness of both intermediate trophoblast and melanoma cell. Shih et al's ${ }^{22}$ and our recent data indicate that CD146 is also involved in the regulation of trophoblast migration and matrix metalloproteinase secretion.

The expression of CD31/PECAM-1 in both normal and pre-eclamptic placentas has been studied by several groups. ${ }^{7,8,24-27}$ However, controversy still exists regarding its distribution. For instance, Zhou et $a l^{7,8}$ reported that invasive cytotrophoblasts in normal placentas expressed CD31/PECAM-1 molecule, whereas it was absent in pre-eclampsia. However, Lyall ${ }^{27}$ showed that CD31/PECAM-1 was undetectable on any trophoblasts in both normal and pre-eclamptic placentas. Here, we tested the CD31/PECAM-1 staining compared with CD146 in normal and pre-eclamptic placentas. Our data showed that CD31/PECAM-1 exhibited negative staining on trophoblasts in both normal pregnancy and pre-eclampsia, which is in agreement with the results reported by Lyall.

In summary, this study provides a number of important insights into the contribution of cell adhesion molecules (especially endothelial CD146 and CD31) underlying normal and disordered pregnancy. First, a highly sensitive and specific CD146 expression on intermediate trophoblast provides a very useful marker for distinguishing and studying three trophoblast subpopulations. Second, the fact that pre-eclamptic trophoblasts fail to express CD146 molecule provides further evidence in understanding the role of CD146 in trophoblast invasion and the pathogenesis of pre-eclampsia. Third, the different expression patterns of CD146 and CD31 we have observed in normal pregnancy and in pre-eclampsia prompt us to further investigate the regulation and function of these molecules in the endothelium and trophoblast.

\section{Acknowledgement}

This work was supported by the National 863- and 973-projects, the National Natural Sciences Foundation of China and CAS Grant.

\section{References}

1 Walker JJ. Pre-eclampsia. Lancet 2000;356:1260-1265.
2 Dekker GA, Sibai BM. Etiology and pathogenesis of preeclampsia: current concepts. Am J Obstet Gynecol 1998;179:1359-1375.

3 Granger JP, Alexander BT, Llinas MT, et al. Pathophysiology of preeclampsia: linking placental ischemia/ hypoxia with microvascular dysfunction. Microcirculation 2002;9:147-160.

4 Kurman RJ. The morphology, biology, and pathology of intermediate trophoblasts: a look back to the present. Hum Pathol 1991;22:847-855.

5 Khong TY, Sawyer IH, Heryet AR. An immunohistologic study of endothelialization of uteroplacental vessels in human pregnancy-evidence that endothelium is focally disrupted by trophoblast in preeclampsia. Am J Obstet Gynecol 1992;167:751-756.

6 Cavallaro U, Christofori G. Cell adhesion in tumor invasion and metastasis: loss of the glue is not enough. Biochim Biophys Acta 2001;1552:39-45.

7 Zhou Y, Fisher SJ, Janatpour M, et al. Human cytotrophoblasts adopt a vascular phenotype as they differentiate. A strategy for successful endovascular invasion? J Clin Invest 1997;99:2139-2151.

8 Zhou Y, Damsky CH, Fisher SJ. Preeclampsia is associated with failure of human cytotrophoblasts to mimic a vascular adhesion phenotype. J Clin Invest 1997;99:2152-2164.

9 Lehmann JM, Riethmuller G, Johnson JP. MUC18, a marker of tumor progression in human melanoma, shows sequence similarity to the neural cell adhesion molecules of the immunoglobulin superfamily. Proc Natl Acad Sci USA 1989;86:9891-9895.

10 Shih IM, Elder DE, Speicher D, et al. Isolation and functional characterization of the A32 melanomaassociated antigens. Cancer Res 1994;54:2514-2520.

11 Bardin N, Frances V, Lesaule G, et al. Identification of the S-Endo 1 endothelial-associated antigen. Biochem Biophys Res Commun 1996;218:210-216.

12 Shih IM, Nesbit M, Herlyn M, et al. A new Mel-CAM (CD146)-specific monoclonal antibody, MN-4, on paraffin-embedded tissue. Mod Pathol 1998;11:10981106.

13 Shih IM, Kurman RJ. Expression of melanoma cell adhesion molecule in intermediate trophoblast. Lab Invest 1996;75:377-388.

14 Pickl WF, Majdic O, Fischer GF, et al. MUC18/MCAM (CD146), an activation antigen of human T lymphocytes. J Immunol 1997;158:2107-2115.

15 Luca M, Hunt B, Bucana CD, et al. Direct correlation between MUC18 expression and metastatic potential of human melanoma cells. Melanoma Res 1993;3: 35-41.

16 Xie S, Luca M, Huang S, et al. Expression of MCAM/ MUC18 by human melanoma cells leads to increased tumor growth and metastasis. Cancer Res 1997;57: 2295-2303.

17 Satyamoorthy K, Muyrers J, Meier F, et al. MelCAM-specific genetic suppressor elements inhibit melanoma growth and invasion through loss of gap junctional communication. Oncogene 2001;20: 4676-4684.

18 Bardin N, Anfosso F, Masse JM, et al. Identification of CD146 as a component of the endothelial junction involved in the control of cell-cell cohesion. Blood 2001;98:3677-3684.

19 Anfosso F, Bardin N, Frances V, et al. Activation of human endothelial cells via S-endo-1 antigen (CD146) stimulates the tyrosine phosphorylation of 
focal adhesion kinase p125(FAK). J Biol Chem 1998;273:26852-26856.

20 Anfosso F, Bardin N, Vivier E, et al. Outside-in signaling pathway linked to CD146 engagement in human endothelial cells. J Biol Chem. 2001;276:1564-1569.

21 Yan X, Lin Y, Yang D, et al. A novel anti-CD146 monoclonal antibody, AA98, inhibits angiogenesis and tumor growth. Blood 2003;102:184-191.

22 Shih I, Wang T, Wu T, et al. Expression of Mel-CAM in implantation site intermediate trophoblastic cell line, IST-1, limits its migration on uterine smooth muscle cells. J Cell Sci 1998;111:2655-2664.

23 Goldman-Wohl D, Yagel S. Regulation of trophoblast invasion: from normal implantation to pre-eclampsia. Mol Cell Endocrinol 2002;187:233-238.

24 Lyall F, Greer IA, Boswell F, et al. Expression of cell-adhesion molecules in placentae from pregnancies complicated by preeclampsia and intrauterine growth retardation. Placenta 1995;16:79-87.

25 Blankenship TN, Enders AC. Expression of plateletendothelial cell adhesion molecule-1 (PECAM) by macaque trophoblast cells during invasion of the spiral arteries. Anat Rec 1997;247:413-419.

26 Coukos G, Makrigiannakis A, Amin K, et al. Platelet endothelial cell adhesion molecule-1 is expressed by a subpopulation of human trophoblasts: a possible mechanism for trophoblast-endothelial interaction during haemochorial placentation. Mol Hum Reprod 1998;4:357-367.

27 Lyall F, Bulmer JN, Duffie E, et al. Human trophoblast invasion and spiral artery transformation: the role of PECAM-1 in normal pregnancy, preeclampsia, and fetal growth restriction. Am J Pathol 2001;158: 1713-1721. 